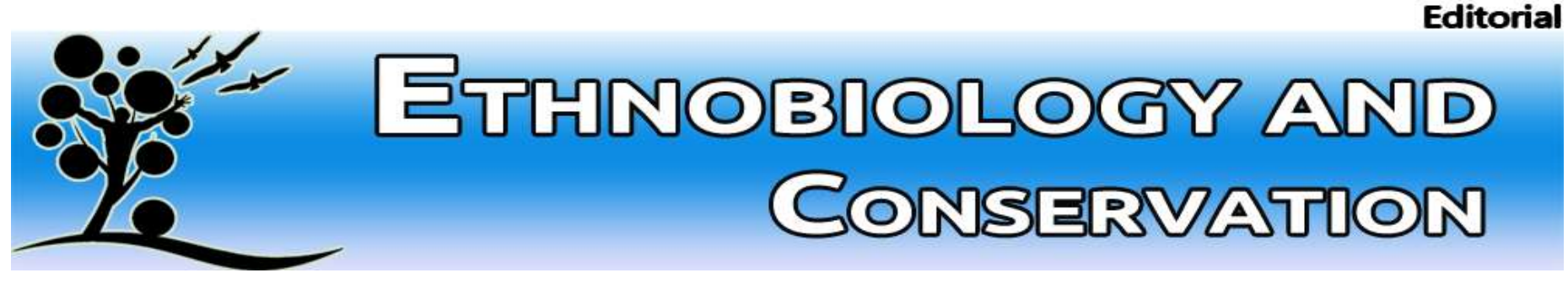

\title{
How to improve the quality of scientific publications in ethnobiology
}

\author{
Ulysses Paulino Albuquerque \\ ${ }^{1}$ Departamento de Biologia, Laboratório de Etnobotânica Aplicada, Universidade Federal Rural de \\ Pernambuco, Recife, Pernambuco, Brazil \\ هE-mail address: upa@db.ufrpe.br
}

Ethnobiology and Conservation 2013, 2:4 (28 May 2013)

ISSN 2238-4782

doi: 10.15451/ec2013-8-2.4-1-05

ethnobioconservation.com

The scientific output in ethnobiology is growing worldwide, particularly in Latin America. This growth has prompted an obvious question: is the quality of these publications growing at the same rate? Assessing the quality of scientific production is not an easy task, although the worldwide adoption of metrics, such as Impact Factor, has become very common (Albuquerque 2010). In the case of impact factor specifically, there is increasing criticism regarding its use to assess the quality of the output from a researcher or institution as a factor in career advancement or to grant research funds. Bruce Alberts (2013), in a recent editorial in Science, rekindled the discussion over the current misuse of the impact factor as a metric.

Independent of any discussion of the proper tools to evaluate scientific production, an evaluation of the progress made by a given science is necessary to determine how to proceed. In 2009, we provided an overview of the problems that occur specifically in the field of ethnobotany (Albuquerque and Hanazaki 2009; see also Albuquerque et al. 2011), and we noted some of the misconceptions and suggest solutions for them. From a broader perspective, it is clear that the difficulties of producing interesting articles extend to the entire field of ethnobiology. Now, we aim to propose some suggestions for ethnobiology base in our ideas (see Albuquerque and Hanazaki 2009).

1. The continued education of ethnobiologists, with emphasis on developing skills in the areas of scientific writing and the methods and philosophy of science.

Many of the weaknesses found in scientific production result from bad science. This phenomenon is not limited to the lack of ability to write in another language (English, the "official" language of science), or of basic knowledge about scientific 
writing, although all of these issues contribute to the final product quality. Many scientists are poorly trained, which is reflected in their work. I have heard the following from various students: "I have to incorporate statistics in my work to make it more scientific." Who asserted that the use of statistics or the use of any quantitative tool is what makes a work scientifically acceptable? In fact, the quality of science depends on the quality of the question that directs the researcher and the use of a solid and appropriate theory that provides a scenario for the research. Moreover, some studies include a variety of statistical tools that are fragile and misguided. Unfortunately, many people confuse quantification with scientific quality, and this distortion has been widely disseminated in the field of ethnobiology, especially in ethnobotany. The problem is confusing the tool (the method) with the science (the research question and hypotheses). We have a lot of publications that replicate methods and protocols, but do not advance scientific knowledge.

In a recent study, our research group used two important articles published in the field of ethnobotany as samples (Ramos et al. 2012). In addition to offering new techniques for data analysis, these articles also discuss new theoretical issues that deserve the attention of scientists. We find that a number of subsequently published articles that cited these studies and used them as a "model" in some way do not mention the same articles for their theoretical contributions. In fact, the subsequent articles only reproduced the suggested techniques uncritically and often with misrepresentation.

In comparison to the rest of the world, Latin America is young in terms of its scientific research production, and ethnobiology, in general, is a relatively new science. In Brazil, over the last 10 years, we have been encouraged to become more aware of the state of science, noting that by definition, science is universal. Even with this forced awakening, concern exists whether our scientists are developing properly, or whether we are even educating scientists. For example, in many scientific fields, Brazil is recognized as a leader in the production of knowledge, as in the case of studies on biodiversity. However, it is also true that this recent awakening has not led us to produce theories that help to explain the observed patterns of biodiversity, or even to detect the existence of these patterns.

The same phenomenon is occurring within the field of ethnobiology. The significant increase in publications, in both local and international journals, prompts another question: Are we in fact advancing as a science? I have observed:

a) Many young researchers believe that a science is defined by its object of study. They forget that science is a particular way of thinking, rather than gathering information on a particular subject. This belief reveals insufficient training and lack of knowledge in the philosophy and methodology of science.

b) Some scientists believe that they can fulfill "the social contract of science," to which all scientists are bound, by publishing anything in any journal, and in any language. This observation reveals a lack of basic knowledge about scientific communication and the intricate and complex mechanisms for the production of scientific knowledge.

\section{The definition of global investigation strategies and lines of action}

This definition is another aspect that I consider to be important. The field of ethnobiology has great theoretical and methodological complexity, which is reflected 
in publications. This complexity is reflected in the different research themes. Such a circumstance is not bad and is even desirable. However, when reviewing ethnobiological literature, it is often lacking direction as well as a prior knowledge of what has been written and discussed by other scientists. I believe that the definition of global strategies, without limiting the freedom of scientists in their areas of interest, can be a healthy way to advance the ethnobiology field.

\section{The elaboration of research agendas based on questions that will advance the field into high priority areas, such as biodiversity conservation}

A science that seeks to mature will require that scientists in the field define their research agenda. The development of more global agendas can align perfectly with the ideas described in the previous topic. We wrote that "these research agendas could be more regional and perhaps associated with the global strategies discussed above. An example of a basic question would ask what type of knowledge is needed to effectively corroborate the strategies directed towards biodiversity conservation or the discovery of new drugs of medical and pharmaceutical interest" (e.g. Albuquerque and Hanazaki 2009). Undoubtedly, ethnobiology today plays important roles, such as those mentioned above, especially relating to the local development and empowerment of communities that are partners in ethnobiological investigations (see also Wolverton 2013).

\section{The construction of standardized methodological protocols to address basic questions, to allow data collected in different regions to be more easily compared and to make the associated results more amenable to generalization}

The adoption of standardized protocols to answer certain questions can undoubtedly move us towards better testing of hypotheses and building theories in ethnobiology. Today, we have great difficulty in making generalizations because of the significant diversity of the methods that researchers use to test similar issues. We must advance not only the incorporation of theory into our work, but must also evaluate the quality and appropriateness of the methods we use. The journal Ethnobiology and Conservation has a dedicated space for researchers to propose hypotheses for study and ways to test these hypotheses (see Ferreira Júnior et al. 2013). As an approach to ethnobotany, "these protocols would need to be exhaustively debated in terms of their methodological and interpretative limits. (...) congresses and meetings should be promoted to discuss these questions among ethnobotanists interested in constructing a dynamic and constantly advancing ethnobotanical science" (Albuquerque and Hanazaki 2009).

\section{What to expect for the future?}

I argue that we should to discuss the advancing of ethnobiological science in depth. What training is needed for young ethnobiologists? What types of knowledge do we need to produce? Reflecting on these themes can help us to move forward and plan the future of ethnobiology. I do not believe that today's problem of low quality scientific production, especially in developing countries, is the result, as some argue, of the lack of access to books, manuals or quality papers. The internet has completely changed this situation. Today, we have access to archived materials from libraries and repositories around the world. Initiatives such as "Open Access" 
democratized access to scientific information. I believe that we cannot move forward in terms of the quality of our investigations while these myths about science and scientific production persist.

Many scientists around the world are arguing that acceleration of scientific knowledge production and the increased requirements to publish on the best journals have led to many researchers not having the time to mature their findings. This predicament results in prematurely publishing information that could wait a little longer for publicity. Surely, this problem affects the quality of scientific production. Theoretically, this information could reach the public when it is more mature, well reasoned, and therefore when it will have a greater impact.

Reinach (2013) critiques the increasingly commonplace practice in which many researchers divide the results of their investigations in several publications to increase the number of papers published. He describes the damage caused by this practice in a speech that echoed through the corridors of Universities and Research Institutes in Brazil:

Nowadays, conversation among post-graduate students and scientists is different at the best Brazilian universities. Most of them are concerned about the amount of studies they published in a year and where they published it. They want to know how they will be classified. "So-and-so is now a $1 B \mathrm{CNPq}$ researcher. "It could not be different given his 8 works published in highimpact journals."'So-and-so's department was lowered to Capes ${ }^{1}$ concept 4. With few thesis last year and only two publications in low-impact journals..." It is not that those people's eyes do not shine when they discuss their researches, but the explanation of how someone published in Nature calls more attention than a new way of approaching a problem considered as unsolvable.

To summarize, I would conclude with the following guidelines:

a) Working slowly is important. Our young scientists must be instructed early in their careers to consider research programs. Thinking of a "research program" implies the need to define what data need to be collected to best answer the questions as well as the need to analyze the theory or theories that must interpret such data. Data that can be published independently and periodically must also be provided.

b) We must not perpetuate the idea that a good scientist or researcher will publish 10 articles per year. It is a distorted and misguided view of science, and scientific practice, to believe that one needs an exaggerated number of publications to stay competitive. Some scientists are more prolific with publishing their work because they divide their findings. Some scientists are more prolific with publishing their work because they have a solid research program through which publishing has become a routine part of their laboratories. Some scientists are more prolific with publishing their work because they submit to journals that lack specific criteria or good quality controls and contain irrelevant topics in order to grow the publication output. Moreover, some scientists are more prolific with publishing their work because they resort to procedures that are ethically questionable (see Albuquerque 2011).

\footnotetext{
${ }^{1}$ CAPES - Brazilian agency responsible for the evaluation of post-graduate courses in Brazil.
} 
The measure of the quality of a researcher or scientist is not the quantity of articles published, but the ideas that follow the responses that the research generates. There are good scientists who publish a lot, and there are good scientists who publish little and regularly. Ultimately, all of these scientists have published! Scientists who have "matured" in terms of their production of scientific knowledge may regularly publish in a specific area of research, regardless of the need for three years to collect all of the data to answer a research question. Therefore, what measures the quality of knowledge produced in a given science is what the scientists are doing in said science, not only to advance the knowledge of our world and ourselves, but also to solve the problems and challenges of our existence on this planet.

\section{Acknowledgements}

To CNPq for the financial support and research productivity scholarship granted to the author.

\section{References}

Alberts B (2013) Impact factor distortions. Science 340(6134): 787.

Albuquerque UP (2010) The tyranny of the impact factor: why do we still want to be subjugated? Rodriguesia 61: 353-358.

Albuquerque UP (2011) Scientific misconduct. WebmedCentral BIOETHICS 2011;2(3):WMC001719.

Albuquerque UP, Hanazaki N (2009) Five problems in current ethnobotanical research - and some suggestions for strengthening them. Human Ecology 37: 653-661.

Albuquerque UP, Ramos MA, Medeiros MFT (2011) Experiences of ethnobotanists with publication: a first approach. Bioscience 61: 706-712.

Ferreira Júnior WS, Santoro FR, Nascimento ALB, Ladio AH, Albuquerque UP (2013) The role of individuals in the resilience of local medical systems based on the use of medicinal plants a hypothesis. Ethnobiology and Conservation 2: 1.

Ramos MA, Melo JG, Albuquerque UP (2012) Citation behavior in popular scientific papers: what is behind obscure citations? The case of ethnobotany. Scientometrics 92: 711-719.

Reinach F (2013) Darwin e a prática da 'Salami Science'. Estadão.com.br/São Paulo. April, 13, 2013.

Wolverton S (2013) Ethnobiology 5: Interdisciplinarity in an era of rapid environmental change. Ethnobiology Letters 4: 21-22. 